\title{
PLEOMORPHIC SKIN ERUPTIONS IN A COVID-19 AFFECTED PATIENT: CASE REPORT AND REVIEW OF THE LITERATURE
}

\author{
Enrico Scala ${ }^{1}$, Luca Fania ${ }^{1}$, Filippo Bernardini ${ }^{1}$, Rodolfo Calarco ${ }^{1}$, Sabrina Chiloiro ${ }^{1}$, \\ Cristiana Di Campli ${ }^{1}$, Sabrina Erculei ${ }^{1}$, Mauro Giani ${ }^{1}$, Marzia Giordano ${ }^{1}$, Annarita \\ Panebianco ${ }^{1}$, Francesca Passarelli ${ }^{1}$, Andrea Trovè ${ }^{1}$, Sofia Verkhovskaia ${ }^{1}$, Giandomenico \\ Russo ${ }^{1}$, Antonio Sgadari ${ }^{2}$, Biagio Didona ${ }^{1}$, and Damiano Abeni ${ }^{1}$ \\ ${ }^{1}$ Istituto Dermopatico dell'Immacolata IRCCS \\ ${ }^{2}$ Istituto Dermopatico dell'Immacolata Istituto di Ricovero e Cura a Carattere Scientifico
}

October 14, 2020

\begin{abstract}
Abstract The coronavirus disease (COVID-19), during its course, may involve several organs, including the skin with a petechial skin rash, urticaria and erythematous rash, or varicella-like eruption, representing an additional effect of the SARS-CoV-2 infection, as commonly observed in other viral diseases. Considering that symptomatic COVID-19 patients generally undergo multi-drug treatments, the occurrence of a possible adverse drug reaction (ADR) presenting with cutaneous manifestations should be contemplated. Pleomorphic skin eruptions occurred in a 59-year-old Caucasian woman, affected by a stable form of Chronic lymphocytic leukaemia, and symptomatic SARS-CoV.2 infection, treated with a combination of hydroxychloroquine sulphate, darunavir, ritonavir, sarilumb, omeprazole, ceftriaxone, high-flow oxygen therapy devices, filgrastim (Zarzioß) as a single injection, and enoxaparin. The patient stopped all treatment but oxygen and enoxaparin and received a high-dose Desametasone with complete remission of dermatological impairment in 10 days. It is very important to differentially diagnose COVID-19 disease-related cutaneous manifestations, where is justified to continue the multi-drug antiviral treatment, from those caused by an adverse drug reaction, where it would be necessary to identify the possible culprit drug and to start an appropriate treatment.
\end{abstract}

\section{Hosted file}

ALL-2020-01318.pdf available at https://authorea.com/users/328414/articles/486721pleomorphic-skin-eruptions-in-a-covid-19-affected-patient-case-report-and-review-ofthe-literature 

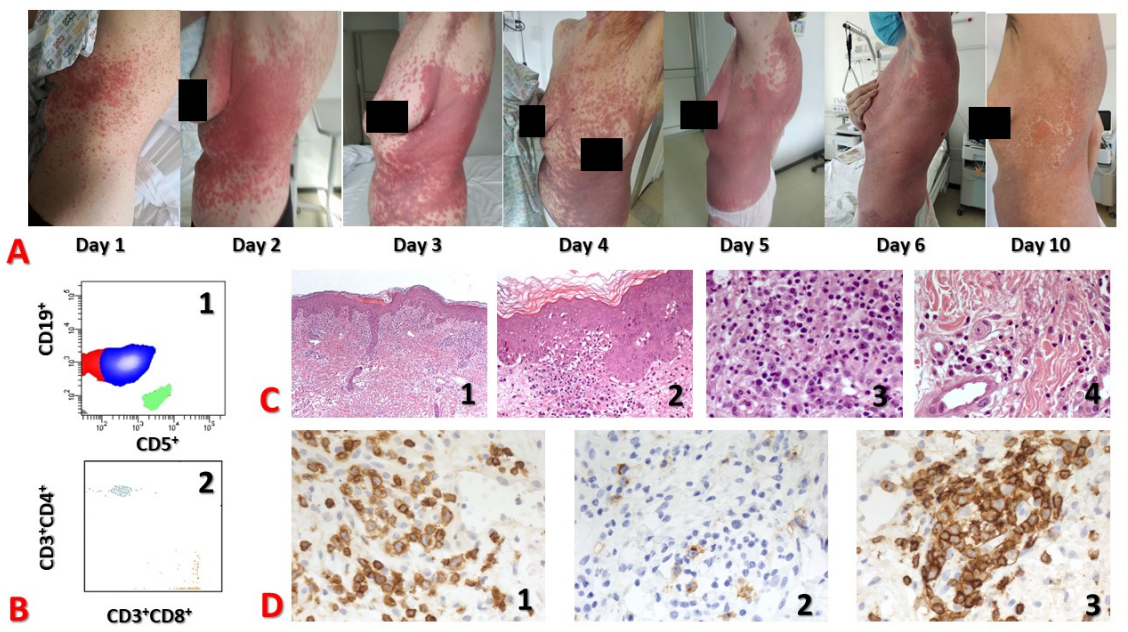